Pacific Journal of Mathematics

ON MAJORANTS FOR SOLUTIONS OF ALGEBRAIC
DEFERENTIAL EQUATIONS IN REGIONS OF THE COMPLEX

SEVEN BARRY BANK 


\title{
ON MAJORANTS FOR SOLUTIONS OF ALGEBRAIC DIFFERENTIAL EQUATIONS IN REGIONS OF THE COMPLEX PLANE
}

\author{
STEVEN BANK
}

\begin{abstract}
In this paper, we investigate the rate of growth of functions which are analytic in an arbitrary simplyconnected region of the complex plane and which are solutions of first order algebraic differential equations (i.e., equations of the form $\Omega(z, y, d y / d z)=0$, where $\Omega$ is a polynomial in $z, y$ and $d y / d z)$.
\end{abstract}

In the course of constructing an example for second order equations, Vijayaraghavan in [5] showed that for any realvalued increasing function $\Phi(x)$ on the interval $(0,+\infty)$, it is possible to find a complex function $h(z)$, which is analytic in a simply-connected region $R$ of the plane containing $(0,+\infty)$, and satisfies a first order algebraic differential equation, and which has the property that $|h(x)|>\Phi(x)$ at a sequence of real $x$ tending to $+\infty^{1}$. For a given $\Phi(x)$, the function $h(z)$ constructed was of the form $P(\alpha z)$ where $P(u)$ is the Weierstrass $P$-function with primitive periods $w$ and $i w^{\prime}\left(w, w^{\prime}\right.$ real), and where the constant $a$ was of the form $a=w+i b$, where $b$ depends on $\Phi$ and $b / w^{\prime}$ is irrational. Since $P(a z)$ has poles at all points $(m w / a)+\left(n i w^{\prime} / a\right)$ where $m$ and $n$ are integers, clearly the region $R$ associated with the solution $h(z)=P(a z)$ depends on $a$ and hence on $\Phi(x)$. A natural question is thus raised, namely, can such examples be constructed where, for all $\Phi(x)$, the simply-connected region $R$ remains the same. That is, does there exist a simply-connected region $R$ containing $(0,+\infty)$ with the property that for any real-valued increasing function $\Phi(x)$ on $(0,+\infty)$, there is a solution $h(z)$, analytic on $R$, of a first order algebraic differential equation, such that $|h(x)|>\Phi(x)$ at a sequence of real $x$ tending to $+\infty$ ? In this paper we answer this question in the negative by proving the following more general result ( $\$ 2$ below): If $R$ is any simply-connected region, then there exists a real-valued continuous function $\Psi(z)$ on $R$ with the property that for any function $h(z)$, analytic on $R$, which satisfies a first order algebraic differential equation, there is a compact set $K$ contained in $R$ such that $|h(z)|<\Psi(z)$ on $R-K$. In the case where $R$ is not the whole plane, we show that $\Psi(z)$ may be taken to be

${ }^{1}$ None of the solutions $h(z)$ constructed by Vijayaraghavan are real-valued on any interval $\left(x_{0},+\infty\right)$. Of course this is in accord with the well-known result of Lindelöf $\left[2 ;\right.$ p. 213] that a real-valued solution on an interval $\left(x_{0},+\infty\right)$ is majorized, on some interval $\left(x_{1},+\infty\right)$, by $\exp (\exp x)$. 
$\exp \left(\exp \left((1-|f(z)|)^{-1}\right)\right)$, where $f$ is a univalent analytic mapping of $R$ onto the unit disk (which exists by the Riemann Mapping Theorem). In the case where $R$ is the whole plane, a wellknown result of Valiron [ $3 ;$ p. 41] states that any entire function satisfying a first order algebraic differential equation is of finite order, so $\Psi(z)$ can be taken to be $\exp (\exp |z|)$ in this case.

To prove our main result (assuming $R$ is not the whole plane), the equation is transformed to the unit disk using $f^{-1}$. The resulting equation no longer has polynomial coefficients, but careful estimates on the growth of the coefficients can be made using certain standard estimates on the growth of the univalent analytic function $f^{-1}$ on the unit disk. Then by using results of Valiron [4;p. 299] concerning certain analytic functions in the unit disk, we obtain (Lemma $\mathrm{C}$ below) the same growth condition on solutions in the unit disk as was obtained in [4; p. 294] in the case where the coefficients were polynomials. Our main result then follows by retransforming back to $R$.

\section{We now state our main result:}

THEOREM. Let $R$ be a simply-connected region of the complex plane which is not the whole plane. Let $f$ be a univalent analytic mapping of $R$ onto the unit disk. (Such a mapping exists by the Riemann Mapping Theorem.) Then, if $h(z)$ is any analytic function on $R$ which is a solution of a first order algebraic differential equation, then there exist a constant $A \geqq 0$ and a compact set $X$ contained in $R$, such that the inequality $|h(z)| \leqq \exp \left((1-|f(z)|)^{-4}\right)$ holds for all $z \in R-X$. (Thus clearly, for each such $h(z)$, there is a compact set $K$ contained in $R$ such that the inequality

$$
\left.|h(z)|<\exp \left(\exp \left((1-|f(z)|)^{-1}\right)\right) \text { holds on } R-K \text {. }\right)
$$

REMARK. If $R$ is the whole plane, then by a result of Valiron [3; p. 41], any analytic function on $R$ (i.e., any entire function) which is a solution of a first order algebraic differential equation is of finite order.

3. Proof of the theorem. Let $h(z)$ be analytic on the simplyconnected region $R$ and be a solution of a first order algebraic differential equation $\Omega\left(z, y, y^{\prime}\right)=0$. We may write $\Omega\left(z, y, y^{\prime}\right)$ in the form,

$$
\Omega\left(z, y, y^{\prime}\right)=\sum H_{k j}(z) y^{k}\left(y^{\prime}\right)^{j}
$$

where the functions $H_{k j}(z)$ are polynomials in $z$. Thus,

$$
\sum H_{k j}(z)(h(z))^{k}\left(h^{\prime}(z)\right)^{j} \equiv 0 \quad \text { on } \quad R .
$$


Let $g$ be the inverse of $f$. Thus $g$ is a univalent analytic mapping of the unit disk onto $R$. Since $h(z)$ is analytic on $R$, clearly the function

$$
\varphi(\zeta)=h(g(\zeta)) \text { is analytic on }|\zeta|<1 \text {. }
$$

Since $\phi^{\prime}(\zeta)=h^{\prime}(g(\zeta)) g^{\prime}(\zeta)$, we have by (2) that $\varphi(\zeta)$ satisfies the relation,

$$
\begin{gathered}
\sum F_{k j}(\zeta)(\varphi(\zeta))^{k}\left(\varphi^{\prime}(\zeta)\right)^{j} \equiv 0 \text { on }|\zeta|<1 \text {, where } \\
F_{k j}(\zeta)=H_{k j}(g(\zeta)) /\left(g^{\prime}(\zeta)\right)^{j} \text { for each }(k, j) .
\end{gathered}
$$

For each $(k, j)$ such that $H_{k j} \not \equiv 0$, let $d(k, j)$ be the degree of the polynomial $H_{k j}$. Define

$$
q=1+\max \left\{j+2 d(k, j): H_{k j} \not \equiv 0\right\}
$$

Thus clearly,

$$
q>0
$$

We now prove,

LEMmA A. There exists a constant $K^{*}>0$ such that on any circle $|\zeta|=r$, where $r \in[0,1)$, we have $\left|F_{k j}(\zeta)\right| \leqq K^{*}(1-r)^{-q}$ for all $(k, j)$.

Proof. Since $g(\zeta)$ is univalent on $|\zeta|<1$, the function $G(\zeta)=$ $(g(\zeta)-g(0)) / g^{\prime}(0)$ is also univalent on $|\zeta|<1$ and $G(0)=0$ while $G^{\prime}(0)=1$. Thus by [1; Th. 17. 4. 7, p. 353], $|G(\zeta)| \leqq r(1-r)^{-2}$ on any circle $|\zeta|=r<1$. Since $\mathrm{r}<1$ and $(1-r)^{2} \leqq 1$, we clearly obtain

$$
|g(\zeta)| \leqq L(1-r)^{-2} \quad \text { on }|\zeta|=r<1
$$

where $L=\left|g^{\prime}(0)\right|+|g(0)|>0$ (since $g^{\prime}(0) \neq 0$ ). Again, since $G(\zeta)$ is univalent on $|\zeta|<1$ and $G(0)=0$ while $G^{\prime}(0)=1$ we have by [1; Th. 17. 4. 6, p. 351] that $(1-r)(1+r)^{-3} \leqq\left|G^{\prime}(\zeta)\right| \leqq(1+r)(1-r)^{-3}$ on any circle $|\zeta|=r<1$. Setting $K_{1}=2\left|g^{\prime}(0)\right|$ and $K_{2}=\left|g^{\prime}(0)\right| / 8$, we have

$$
\begin{gathered}
\left.K_{1}>0 \text { and } K_{2}>0 \text { (since } g^{\prime}(0) \neq 0\right) \text {, and } \\
K_{2}(1-r) \leqq\left|g^{\prime}(\zeta)\right| \leqq K_{1}(1-r)^{-3} \text { on }|\zeta|=r<1 .
\end{gathered}
$$

Now consider any $(k, j)$. If $H_{k j} \equiv 0$ clearly $F_{k j} \equiv 0$ by (5), so we may assume $H_{k j} \not \equiv 0$. Since $H_{k j}$ is of degree $d=d(k, j)$, we may write, $H_{k j}(z)=B_{0}+B_{1} z+\cdots+B_{d} z^{d}$ where the $B_{j}$ are constants. Thus by (8), we have on any circle $|\zeta|=r<1$, 


$$
\left|H_{k j}(g(\zeta))\right| \leqq\left|B_{0}\right|+\left|B_{1}\right| L(1-r)^{-2}+\cdots+\left|B_{d}\right| L^{d}(1-r)^{-2 d} .
$$

Thus clearly there is a constant $\alpha(k, j)>0$ such that,

$$
\left|H_{k j}(g(\zeta))\right| \leqq \alpha(k, j)(1-r)^{-2 d} \text { on }|\zeta|=r<1 \text {. }
$$

By (10), we have $\left|g^{\prime}(\zeta)\right|^{j} \geqq K_{2}^{j}(1-r)^{j}$ on $|\zeta|=r<1$, so by (12) and (5),

$$
\left|F_{k j}(\zeta)\right| \leqq\left(\alpha(k, j) / K_{2}^{j}\right)(1-r)^{-(j+2 d)} \text { on } \quad|\zeta|=r<1 \text {. }
$$

Setting

$$
K^{*}=1+\max \left\{\alpha(k, j) / K_{2}^{j}: H_{k j} \not \equiv 0\right\}
$$

and noting that $q>j+2 d(k, j)$ by (6), we have by (13) that for any $(k, j)$

$$
\left|F_{k j}(\zeta)\right| \leqq K^{*}(1-r)^{-q} \text { on any circle }|\zeta|=r^{*}<1,
$$

proving Lemma A.

Now set

$$
\begin{gathered}
p=\max \left\{k+j: H_{k j} \not \equiv 0\right\}, \quad \text { and } \\
m=\max \left\{j: H_{p-j, j} \not \equiv 0\right\} .
\end{gathered}
$$

We now prove,

Lemma B. There exist constants $K^{*}>0, \sigma \geqq 0$ and $r_{0} \in[0,1)$ such that on any circle $|\zeta|=r$, where $r_{0}<r<1$, we have $\left|F_{p-m, m}(\zeta)\right| \geqq K^{\sharp}\left(1-\gamma^{\circ}\right)^{\sigma}$.

Proof. If the degree $d$ of $H_{p-m, m}$ is zero, then $H_{p-m, m}$ is a nonzero constant function, say $H_{p-m, m}(z) \equiv L_{1}$. Then by (5) and (10), we have $\left|F_{p-m},,_{m}(\zeta)\right| \geqq\left(\left|L_{1}\right| / K_{1}^{m}\right)\left(1-r^{3 m}\right.$ on $|\zeta|=r<1$ which proves the lemma in this case.

Thus we may assume $d>0$. Since $H_{p-m, m}(z)$ is a polynomial of degree $d \geqq 1$, we may write,

$$
H_{p-m, m}(z)=B\left(z-a_{1}\right) \cdots\left(z-a_{d}\right) \text { where } B \neq 0,
$$

and hence

$$
H_{p-m, m}(g(\zeta))=B\left(g(\zeta)-a_{1}\right) \cdots\left(g(\zeta)-a_{d}\right) \text { for }|\zeta|<1 .
$$

We partition the set of roots $\left\{a_{1}, \cdots, a_{d}\right\}$ of $H_{p-m, m}(z)$ into three classes and arrange them as follows: $a_{1}, \cdots, a_{b}$ lie in the complement of the closure of $R ; a_{b+1}, \cdots, a_{c}$ lie in $R$ and $a_{c+1}, \cdots, a_{d}$ lie on the boundary of $R$, for some $b$ and $c$ with $0 \leqq b \leqq c \leqq d$. 
We first consider any root $a_{k}, 1 \leqq k \leqq b$. Since $a_{k}$ lies in the complement of the closure of $R$, the distance $\varepsilon_{k}$ from $a_{k}$ to $R$ is strictly positive. But if $|\zeta|<1$ then $g(\zeta)$ lies in $R$ so we have,

$$
\left|g(\zeta)-a_{k}\right| \geqq \varepsilon_{k}>0 \text { for }|\zeta|<1 \text { and } k \in\{1, \cdots, b\} \text {. }
$$

We now consider any root $a_{j}, b+1 \leqq j \leqq c$. Thus $a_{j} \in R$ so there exists $t_{j}$ in the unit disk $|\zeta|<1$ such that $g\left(t_{j}\right)=a_{j}$. Let $\delta=\max \left\{\left|t_{j}\right|: b+1 \leqq j \leqq c\right\}$ and let $r_{0}=(1+\delta) / 2$. Thus $\delta<r_{0}<1$. Let $N$ be the image under $g$ of the annulus $r_{0} \leqq|\zeta|<1$. Then no $a_{j}$ (for $b+1 \leqq j \leqq c$ ) can lie in the closure of $N$, for otherwise there would be a sequence $\left\{\zeta_{n}\right\}$ with $r_{0} \leqq\left|\zeta_{n}\right|<1$ such that $\left\{g\left(\zeta_{n}\right)\right\} \rightarrow a_{j}$. By the continuity of $g^{-1}$ we would obtain $\left\{\zeta_{n}\right\} \rightarrow t_{j}$. Since $\left|\zeta_{n}\right| \geqq r_{0}$ for all $n$, we would then have $\left|t_{j}\right| \geqq r_{0}$ which contradicts $\left|t_{j}\right| \leqq \delta$ (since $\delta<r_{0}$ ). Thus the distance $\varepsilon_{j}$ from $a_{j}$ to $N$ is strictly positive for $j=b+1, \cdots, c$. Thus for $j \in\{b+1, \cdots, c\}$ we have

$$
\left|g(\zeta)-a_{j}\right| \geqq \varepsilon_{j}>0 \text { for } r_{0} \leqq|\zeta|<1 .
$$

Finally, we consider any root $a_{s}, c+1 \leqq s \leqq d$, and we consider the function $G_{s}(\zeta)=\left(g(\zeta)-a_{s}\right)^{-1}$ defined on $|\zeta|<1$. Since $a_{s}$ lies on the boundary of $R$, the equation $g(\zeta)=a_{s}$ has no roots in $|\zeta|<1$ by definition of the map $g(\zeta)$. Thus $G_{s}(\zeta)$ is analytic on $|\zeta|<1$ and is univalent on $|\zeta|<1$ since $g(\zeta)$ is. Thus the function

$$
\psi_{s}(\zeta)=\left(G_{s}(\zeta)-G_{s}(0)\right) / G_{s}^{\prime}(0)
$$

is univalent and analytic on $|\zeta|<1$ and $\psi_{s}(0)=0$ while $\psi_{s}^{\prime}(0)=1$. Thus by [1; Th. 17.4.6, p. 351], we have $\left|\psi_{s}^{\prime}(\zeta)\right| \leqq(1+r)(1-r)^{-3}$ on any circle $\zeta \mid=r<1$. Hence $\left|G_{s}^{\prime}(\zeta)\right| \leqq 2\left|G_{s}^{\prime}(0)\right|(1-r)^{-3}$ on $|\zeta|=$ $r<1$. But $G_{s}^{\prime}(\zeta)=-g^{\prime}(\zeta)\left(g(\zeta)-a_{s}\right)^{-2}$, so we obtain,

$$
\left|g(\zeta)-a_{s}\right|^{2} \geqq\left(1-r^{3}\right)^{\prime}(\zeta)|/ 2| G_{s}^{\prime}(0) \mid \text { on }|\zeta|=r<1 \text {. }
$$

But by $(10),\left|g^{\prime}(\zeta)\right| \geqq K_{2}(1-r)$ on $|\zeta|=r<1$, where $K_{2}>0$. Thus clearly,

$$
\left|g(\zeta)-a_{s}\right|^{2} \geqq\left(K_{2} / 2\left|G_{s}^{\prime}(0)\right|\right)(1-r)^{4} \text { on }|\zeta|=r<1 .
$$

Setting $M_{s}=\left(K_{2} / 2\left|G_{s}^{\prime}(0)\right|\right)^{1 / 2}$, we have $M_{s}>0$ and

$$
\left|g(\xi)-a_{s}\right| \geqq M_{s}(1-r)^{2} \quad \text { on }|\zeta|=r<1 \text { for } s \in\{c+1, \cdots, d\} \text {. }
$$

Thus in view of (18), (19), (20) and (23), we obtain

$$
\left|H_{p-m, m}(g(\zeta))\right| \geqq K_{0}(1-r)^{2(d-c)} \text { on }|\zeta|=r \text {, }
$$

when

$$
r_{0} \leqq r<1 \text {, where } K_{0}=|B| \varepsilon_{1} \cdots \varepsilon_{c} M_{c+1} \cdots M_{d}>0 \text {. }
$$


Now by $(10),\left|g^{\prime}(\zeta)\right|^{m} \leqq K_{1}^{m}(1-r)^{-3 m}$ on $|\zeta|=r<1$, so in view of (5) and (24), we obtain

$$
\left|F_{p-m, m}(\zeta)\right| \geqq K^{\sharp}(1-r)^{\sigma} \quad \text { on } \quad|\zeta|=r \text {, when } \quad r_{0} \leqq r<1 \text {, }
$$

where $K^{\sharp}=K_{0} / K_{1}^{m}>0$ and $\sigma=2(d-c)+3 m \geqq 0$. This proves Lemma $B$.

Now $\varphi(\zeta)$ satisfies the relation (4) in $|\zeta|<1$. We introduce the notation $M(r, \varphi)=\max _{|\zeta|=r}|\varphi(\zeta)|$ for $r \in[0,1)$. We now prove,

Lemma C. There exist real numbers $A \geqq 0$ and $r^{*} \in[0,1)$ such that for any $r \in\left[r^{*}, 1\right)$, we have $M(r, \phi) \leqq \exp \left((1-r)^{-A}\right)$.

Proof. If $M(r, \varphi)$ is bounded on $[0,1)$, then clearly the result holds. Hence we may assume $M(r, \varphi)$ is unbounded so $M(r, \phi) \rightarrow+\infty$ as $r \rightarrow 1$.

We proceed by contradiction and assume the lemma is false. Then in the terminology of [4; p. 294], $\varphi(\zeta)$ is of order $\infty$ in $|\zeta|<1$. Let $\sum_{j=0}^{\infty} c_{j} \zeta^{j}$ be the power series expansion of $\varphi(\zeta)$. For each $r \in[0,1)$, Let $N(r)=\max _{j \geq 0}\left|c_{j}\right| r^{j}$ and $n(r)=\max \left\{j:\left|c_{j}\right| r^{j}=N(r)\right\}$. Then since $\varphi(\zeta)$ is of order $\infty$, by [4; p. 299], for any $b \in(0,1)$, there is in $(0,1)$ a sequence of values of $r$ (called remarkable) tending to one, such that

$$
\log M(r, \varphi)>\gamma^{\prime}(n(r))^{b} \quad \text { and } \quad n(r)>\gamma^{\prime \prime}(1-r)^{-\delta}
$$

where $\delta=(1-b)^{-1}$ and $\gamma^{\prime}, \gamma^{\prime \prime}$ are strictly positive constants independent of $r$, and such that at every point of $|\zeta|=r$ at which $|\varphi(\zeta)|=M(r, \varphi)$ we have

$$
\phi^{\prime}(\zeta)=(1+\epsilon(\zeta))(n(r) / \zeta) \varphi(\zeta)
$$

where $\varepsilon(\zeta)$ tends uniformly to zero as $r=|\zeta|$ tends to one.

Let $q$ be as in (6), so $q>0$ by (7). Let $\sigma$ be as in Lemma B, so $\sigma \geqq 0$. Thus $q+\sigma>0$, so $b=(q+\sigma) /(q+\sigma+1)$ belongs to $(0,1)$. It is for this value of $b$ that we apply the Valiron theory (26), (27). Then the corresponding $\delta=q+\sigma+1$, so

$$
\delta>q+\sigma>0 .
$$

Let $p$ and $m$ be as in (15) and (16). Then clearly by (5),

$$
p=\max \left\{k+j: F_{k j} \not \equiv 0\right\} \text { and } m=\max \left\{j: F_{p-j, j} \not \equiv 0\right\} \text {. }
$$

Now let $r \in(0,1)$ and let $\zeta$ be a point on $|\zeta|=r$ at which $|\varphi(\zeta)|=M(r, \varphi)$. Then $\varphi(\zeta) \neq 0$ and so by dividing (4) through by 
$(\varphi(\zeta))^{p}$ (and using (29)), we can write (4) as

$$
\sum_{j=0}^{m} F_{p-j, j}(\zeta)\left(\varphi^{\prime}(\zeta) / \varphi(\zeta)\right)^{j} \equiv-\sum_{k+j<p} F_{k j}(\zeta)\left(\varphi^{\prime}(\zeta) / \varphi(\zeta)\right)^{j}(\varphi(\zeta))^{k+j-p} .
$$

We will denote the left side of (30) by $\Lambda(\zeta)$, and the right side by $\Gamma(\zeta)$.

We now assert that there exist real numbers $L^{*}>0$ and $r^{*} \in(1 / 2,1)$ such that if $r \in\left(r^{*}, 1\right)$ is a remarkable value, then

$$
|\Gamma(\zeta)| \leqq L^{*}(1-r)^{-q}(M(r, \varphi))^{-1 / 2}
$$

for any point on $|\zeta|=r$ at which $|\varphi(\zeta)|=M(r, \varphi)$.

To prove (31), we note first that for remarkable $r \rightarrow 1$, we have $n(r) \rightarrow+\infty$ by (26). Thus since $M(r, \varphi) \rightarrow+\infty$ and $\varepsilon(\zeta) \rightarrow 0$ for remarkable $r=|\zeta| \rightarrow 1$, we can choose $r^{*} \in(1 / 2,1)$ such that for remarkable $r \in\left(r^{*}, 1\right)$, we have $M(r, \varphi)>1, n(r)>1$ and $|\varepsilon(\zeta)|<1 / 2$ whenever $|\zeta|=r$ and $|\varphi(\zeta)|=M(r, \varphi)$. Now let $r \in\left(r^{*}, 1\right)$ be a remarkable value and let $\zeta$ be a point on $|\zeta|=r$ at which $|\varphi(\zeta)|=$ $M(r, \varphi)$. We refer to the right side of $(30)$. If $k+j<p$, then $\mathrm{p}-(k+j) \geqq 1$, so

$$
|\varphi(\zeta)|^{k+j-p} \leqq(M(r, \varphi))^{-1} .
$$

Since $|\varepsilon(\zeta)|<1 / 2$ and $|\zeta|=r>1 / 2$, we have by (27) that

$$
\left|\varphi^{\prime}(\zeta) / \varphi(\zeta)\right| \leqq 4 n(r) \text {. }
$$

By Lemma A, there is a constant $K^{*}>0$ such that $\left|F_{k j}(\zeta)\right| \leqq$ $K^{*}(1-r)^{-q}$ for all $(k, j)$. Thus by $(32)$, (33) and the definition of $\Gamma(\zeta)$, it follows that

$$
|\Gamma(\zeta)| \leqq K_{3}(1-r)^{-q}(n(r))^{g}(M(r, \varphi))^{-1}
$$

where $K_{3}>0$ and $\theta \geqq 0$ are constants (independent of $r$ ). But by (26), $\gamma^{\prime}(n(r))^{b}<\log M(r, \varphi)$, so by (34),

$$
|\Gamma(\zeta)| \leqq L^{*}(1-r)^{-q}(\log M(r, \varphi))^{9 / b}(M(r, \varphi))^{-1}
$$

where $L^{*}>0$ is a constant (independent of $r$ ). But since $M(r, \varphi) \rightarrow+\infty$ as $r \rightarrow 1$, clearly there exists $r^{\prime} \in(0,1)$ such that if $r \in\left(r^{\prime}, 1\right)$, then $(\log M(r, \varphi))^{\theta / b}<(M(r, \varphi))^{1 / 2}$. Setting $r^{\sharp}=\max \left\{r^{*}, r^{\prime}\right\}$, we see that (31) follows from (35).

We now consider $\Lambda(\zeta)=\sum_{j=0}^{m} F_{p-j, j}(\zeta)\left(\phi^{\prime}(\zeta) / \varphi(\zeta)\right)^{j}$.

Case I. $m=0$. Then $\Lambda(\zeta)=F_{p-m, m}(\zeta)$. By Lemma B there exist constants $K^{\sharp}>0$ and $r_{0} \in\left(r^{\sharp}, 1\right)$, such that on any circle $|\zeta|=r$ where $r \in\left(r_{0}, 1\right)$, we have 


$$
|\Lambda(\zeta)|=\left|F_{p-m, m}(\zeta)\right| \geqq K^{\sharp}(1-r)^{\sigma} .
$$

Since $\Lambda(\zeta)=\Gamma(\zeta)$ (by (30)), we have by (31) and (36) that for all remarkable $r \in\left(r_{0}, 1\right), K^{\sharp}(1-r)^{\sigma} \leqq L^{*}(1-\mathrm{r})^{-q}\left(M(r, \varphi)^{-1 / 2}\right.$ and hence

$$
(1-r)^{2(q+\sigma)} M(r, \varphi) \leqq\left(L^{*} / K^{\sharp}\right)^{2} .
$$

But by (26), there exist constants $\gamma^{\prime}>0, \gamma^{\prime \prime}>0$, independent of $r$, such that $\log M(r, \varphi)>\gamma^{\prime}\left(\gamma^{\prime \prime}\right)^{b}(1-r)^{-b \delta}$. Hence with (37), we see that for all remarkable $r \in\left(r_{0}, 1\right)$,

$$
(1-r)^{2(q+\sigma)} \exp \left(\gamma(1-r)^{-b \delta}\right) \leqq\left(L^{*} / K^{\ddagger}\right)^{2}
$$

where $\gamma=\gamma^{\prime}\left(\gamma^{\prime \prime}\right)^{b}>0$. Since $b \delta=q+\sigma>0$ (by (28)), it is clear that (38) is impossible since the left side of (38) tends to $+\infty$ as $r \rightarrow 1$. This contradiction proves Lemma $\mathrm{C}$ in the case $m=0$.

Case II. $m>0$. Since $\varepsilon(\zeta)$ (in (27)) tends to zero as $r=|\zeta| \rightarrow 1$, we can choose $r_{1} \in(0,1)$ such that for any remarkable $r \in\left(r_{1}, 1\right)$, we have $|\varepsilon(\zeta)|<1 / 2$ for those points $\zeta$ on $|\zeta|=r$ at which $|\varphi(\zeta)|=$ $M(r, \varphi)$. Hence for those $\zeta$, we have by $(27)$,

$$
\left|\zeta \varphi^{\prime}(\zeta) / n(r) \varphi(\zeta)\right| \geqq 1-|\varepsilon(\zeta)|>1 / 2 \text {. }
$$

Since $|\zeta|<1$, we have $\left|\varphi^{\prime}(\zeta) / \varphi(\zeta)\right|>n(r) / 2$ and so by (26), we have

$$
\left|\varphi^{\prime}(\zeta) / \varphi(\zeta)\right|>\left(\gamma^{\prime \prime} / 2\right)(1-r)^{-\delta} \quad \text { where } \quad \gamma^{\prime \prime}>0 \text {. }
$$

We now assert that there exists a constant $K_{0}>0$, such that for all sufficiently large remarkable $r$, we have

$$
|\Lambda(\zeta)| \geqq K_{0}\left(1-r^{\sigma}\right)^{\sigma-m \tilde{\jmath}}
$$

at all points of $|\zeta|=r$ at which $|\varphi(\zeta)|=M(r, \varphi)$.

To prove (40), we consider the quotients,

$$
Q_{j}(\zeta)=\left(F_{p-j, j}(\zeta)\left(\varphi^{\prime}(\zeta) / \varphi(\zeta)\right)^{j}\right) /\left(F_{p-m, m}(\zeta)\left(\varphi^{\prime}(\zeta) / \varphi(\zeta)\right)^{m}\right),
$$

for $j=0,1, \cdots, m-1$. By Lemma A, there is a constant $K^{*}>0$ such that $\left|F_{p-j, j}(\zeta)\right| \leqq K^{*}(1-r)^{-q}$ on any circle $|\zeta|=r<1$, for all $j$, while by Lemma $\mathrm{B}$, there is a constant $K^{*}>0$ such that for all sufficiently large $r \in(0,1)$, we have

$$
\left|F_{p-m, m}(\zeta)\right| \geqq K^{\sharp}(1-r)^{\sigma} \quad \text { on } \quad|\zeta|=r \text {. }
$$

Thus, with (39), we see that for $j=0,1, \cdots, m-1$, there is a constant $L_{j}>0$ such that

$$
\left|Q_{j}(\zeta)\right| \leqq L_{j}(1-r)^{(m-j) \hat{o}-(q+\sigma)}
$$


for all sufficiently large remarkable $r$ and all points $\zeta$ on $|\zeta|=r$ at which $|\varphi(\zeta)|=M(r, \varphi)$. Since $(m-j) \delta \geqq \delta>q+\sigma$ (by (28)), we see that the right side of (42) tends to zero as $r \rightarrow 1$, so for all sufficiently large remarkable $r$, we have

$$
\left|Q_{j}(\zeta)\right| \leqq 1 /(m+1) \text { for } j=0,1, \cdots, m-1,
$$

at all points on $|\zeta|=r$ at which $|\varphi(\zeta)|=M(r, \varphi)$. Now by definition of $\Lambda$, we have

$$
\Lambda(\zeta)=F_{p-m, m}(\zeta)\left(\phi^{\prime}(\zeta) / \varphi(\zeta)\right)^{m}\left(1+\sum_{j=0}^{m-1} Q_{j}(\zeta)\right),
$$

and so by (43), we have

$$
|\Lambda(\zeta)| \geqq(1 /(m+1)) \mid F_{p-m, m}(\zeta)\left(\varphi^{\prime}(\zeta) / \varphi(\zeta)\right)^{m} !
$$

for all sufficiently large remarkable $r$ and all points $\zeta$ on $|\zeta|=r$ at which $|\varphi(\zeta)|=M\left(r^{\circ}, \varphi\right)$. Now it is clear that (40) follows from (39), (41) and (44).

Since $\Gamma(\zeta)=\Lambda(\zeta)$ (by (30)), we have by (31) and (40) that for all sufficiently large remarkable $r, K_{0}(1-r)^{\sigma-m s} \leqq L^{*}(1-r)^{-q}(M(r, \varphi))^{-1 / 2}$, and so

$$
(1-r)^{2(q+\sigma-m \hat{o})} M(r, \varphi) \leqq\left(L^{*} / K_{0}\right)^{2} .
$$

But by $(26), \log M(r, \varphi)>\gamma^{\prime}\left(\gamma^{\prime \prime}\right)^{b}(1-r)^{-b \bar{o}}$ (where $\gamma^{\prime}, \gamma^{\prime \prime}>0$ ), and so for all sufficiently large remarkable $r$, we have

$$
\left(1-r^{2}\right)^{2(q+\sigma-m \bar{c})} \exp \left(\gamma(1-r)^{-b \tilde{o}}\right) \leqq\left(L^{*} / K_{\circ}\right)^{2}
$$

where $\gamma=\gamma^{\prime}\left(\gamma^{\prime \prime}\right)^{b}>0$. Since $b \delta=q+\sigma>0$ (by (28)), it is clear that (46) is impossible, since the left side of (46) tends to $+\infty$ as $r \rightarrow 1$. This contradiction proves Lemma $\mathrm{C}$ in Case II, and thus the proof of Lemma $\mathrm{C}$ is complete.

We now conclude the proof of the theorem. By Lemma C, there exist constants $A \geqq 0$ and $r^{*} \in[0,1)$, such that if $r \in\left[r^{*}, 1\right)$, then

$$
|\varphi(\zeta)| \leqq \exp \left((1-r)^{-A}\right) \quad \text { on } \quad|\zeta|=r<1 \text {. }
$$

Let $X$ be the image under $g$ of the closed disk $|\zeta| \leqq r^{*}$. Then $X$ is a compact set contained in $R$. If $z \in R-X$, then we have $r^{*}<|f(z)|<1$ (since $f=g^{-1}$ ), and so by (47), we have

$$
|\varphi(f(z))| \leqq \exp \left((1-|f(z)|)^{-A}\right) .
$$

But $\varphi=h^{\circ} g$ (by (3)) and so $h(z)=\varphi(f(z))$. Thus 


$$
|h(z)| \leqq \exp \left((1-|f(z)|)^{-A}\right)
$$

if $z \in R-X$, which concludes the proof of the theorem.

\section{BIBLIOGRAPHY}

1. E. Hille, Analytic function theory, Volume II, Ginn and Company, Boston, 1962.

2. E. Lindelöf, Sur la croissance des intégrales des équations différentielles algébrique du premier ordre, Bull. Soc. Math. France 27 (1899), 205-215.

3. G. Valiron, Sur les fonctions entières vérifiant une class d'équations différentielles, Bull. Soc. Math. France 51 (1923), 33-45.

4. - Fonctions analytiques et équations différentielles, J. Math. Pures et. Appl. 31 (1952), 293-303.

5. T. Vijayaraghavan, Sur la croissance des fonctions définies par les équations differentielles, C. R. Acad. Sci. Paris 194 (1932), 827-829.

Received May 13, 1968. This research was supported in part by the National Science Foundation (GP 7374).

UNIVERSITY OF ILLINOIS

URBANA, ILlinoIS 


\title{
PACIFIC JOURNAL OF MATHEMATICS
}

\author{
EDITORS
}

\author{
H. ROYDEN \\ Stanford University \\ Stanford, California \\ RICHARD PIERCE \\ University of Washington \\ Seattle, Washington 98105
}

\author{
J. DUGUNDJI \\ Department of Mathematics \\ University of Southern California \\ Los Angeles, California 90007 \\ BASIL GORDON \\ University of California \\ Los Angeles, California 90024
}

\section{ASSOCIATE EDITORS}

E. F. BECKENBACH
B. H. NeumanN

F. WOLF
K. YoshidA

\section{SUPPORTING INSTITUTIONS}

\author{
UNIVERSITY OF BRITISH COLUMBIA \\ CALIFORNIA INSTITUTE OF TECHNOLOGY \\ UNIVERSITY OF CALIFORNIA \\ MONTANA STATE UNIVERSITY \\ UNIVERSITY OF NEVADA \\ NEW MEXICO STATE UNIVERSITY \\ OREGON STATE UNIVERSITY \\ UNIVERSITY OF OREGON \\ OSAKA UNIVERSITY \\ UNIVERSITY OF SOUTHERN CALIFORNIA
}

\author{
STANFORD UNIVERSITY \\ UNIVERSITY OF TOKYO \\ UNIVERSITY OF UTAH \\ WASHINGTON STATE UNIVERSITY \\ UNIVERSITY OF WASHINGTON

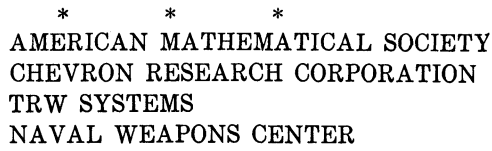

The Supporting Institutions listed above contribute to the cost of publication of this Journal, but they are not owners or publishers and have no responsibility for its content or policies.

Mathematical papers intended for publication in the Pacific Journal of Mathematics should be in typed form or offset-reproduced, double spaced with large margins. Underline Greek letters in red, German in green, and script in blue. The first paragraph or two must be capable of being used separately as a synopsis of the entire paper. It should not contain references to the bibliography. Manuscripts, in duplicate if possible, may be sent to any one of the four editors. Please classify according to the scheme of Math. Rev. 36, 1539-1546. All other communications to the editors should be addressed to the managing editor, Richard Arens, University of California, Los Angeles, California, 90024.

50 reprints are provided free for each article; additional copies may be obtained at cost in multiples of 50 .

The Pacific Journal of Mathematics is published monthly. Effective with Volume 16 the price per volume (3 numbers) is $\$ 8.00$; single issues, $\$ 3.00$. Special price for current issues to individual faculty members of supporting institutions and to individual members of the American Mathematical Society: $\$ 4.00$ per volume; single issues $\$ 1.50$. Back numbers are available.

Subscriptions, orders for back numbers, and changes of address should be sent to Pacific Journal of Mathematics, 103 Highland Boulevard, Berkeley, California, 94708.

PUBLISHED BY PACIFIC JOURNAL OF MATHEMATICS, A NON-PROFIT CORPORATION

Printed at Kokusai Bunken Insatsusha (International Academic Printing Co., Ltd.), 7-17, Fujimi 2-chome, Chiyoda-ku, Tokyo, Japan. 


\section{Pacific Journal of Mathematics}

\section{Vol. 31, No. $3 \quad$ BadMonth, 1969}

George E. Andrews, On a calculus of partition functions .................. 555

Silvio Aurora, A representation theorem for certain connected rings ............ 563

Lawrence Wasson Baggett, A note on groups with finite dual spaces ............. 569

Steven Barry Bank, On majorants for solutions of algebraic differential equations in regions of the complex plane ............................... 573

Klaus R. Bichteler, Locally compact topologies on a group and the corresponding continuous irreducible representations ......................... 583

Mario Borelli, Affine complements of divisors ....................... 595

Carlos Jorge Do Rego Borges, A study of absolute extensor spaces ............. 609

Bruce Langworthy Chalmers, Subspace kernels and minimum problems in Hilbert

spaces with kernel function ...................................... 619

John Dauns, Representation of L-groups and F-rings................. 629

Spencer Ernest Dickson and Kent Ralph Fuller, Algebras for which every

indecomposable right module is invariant in its injective envelope ...........

Robert Fraser and Sam Bernard Nadler, Jr., Sequences of contractive maps and fixed

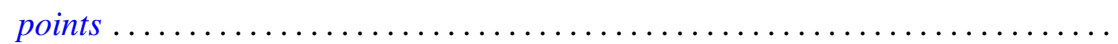

Judith Lee Gersting, A rate of growth criterion for universality of regressive

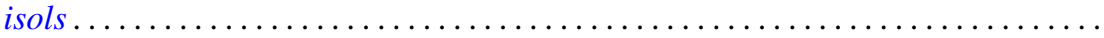

Robert Fred Gordon, Rings in which minimal left ideals are projective ............

Fred Gross, Entire functions of several variables with algebraic derivatives at certain algebraic points

W. Charles (Wilbur) Holland Jr. and Stephen H. McCleary, Wreath products of ordered permutation groups .........................

W. J. Kim, The Schwarzian derivative and multivalence .................. 717

Robert Hamor La Grange, Jr., On $(\mathrm{m}-\mathrm{n})$ products of Boolean algebras ......... 725

Charles D. Masiello, The average of a gauge ........................ 733

Stephen H. McCleary, The closed prime subgroups of certain ordered permutation

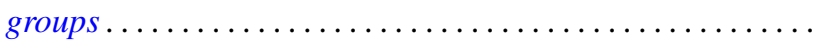

Richard Roy Miller, Gleason parts and Choquet boundary points in convolution measure algebras ...............................

Harold L. Peterson, Jr., On dyadic subspaces ........................ 773

Derek J. S. Robinson, Groups which are minimal with respect to normality being intransitive........................................... 777

Ralph Edwin Showalter, Partial differential equations of Sobolev-Galpern type . . . 787

David Slepian, The content of some extreme simplexes ................... 795

Joseph L. Taylor, Noncommutative convolution measure algebras ............. 809

B. S. Yadav, Contractions of functions and their Fourier series ............... 827

Lindsay Nathan Childs and Frank Rimi DeMeyer, Correction to automorphisms of separable algebras" ....................... 833

Moses Glasner and Richard Emanuel Katz, Correction to: "Function-theoretic degeneracy criteria for Riemannian manifolds".............

Satish Shirali, Correction to: "On the Jordan structure of complex Banach

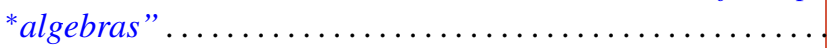

